Colás-Bravo, P. (2021). Retos de la Investigación Educativa tras la pandemia COVID-19. Revista de Investigación Educativa, 39(2), 319-333.

DOI: http://dx.doi.org/10.6018/rie.469871

\title{
Retos de la Investigación Educativa tras la pandemia COVID-19!
}

\section{Challenges of Educational Research after the COVID-19 Pandemic}

\author{
Ma Pilar Colás-Bravo \\ Universidad de Sevilla
}

\begin{abstract}
Resumen
Este texto aporta una visión panorámica sobre los retos de la investigación educativa en un contexto marcado por la pandemia COVID 19 a nivel internacional. Está basado en la lección magistral que se impartió con motivo del Premio Iberoamericano a la Excelencia en Investigación Educativa de 2020, otorgado a la autora de este artículo. El análisis de los efectos sociales, económicos, sanitarios y educativos de la pandemia COVID 19 sirve como punto de referencia para marcar posibles trayectorias científicas por las que debe transitar la investigación educativa. Se marcan como espacios de interés para nuevos estudios las TIC y la educación, el desarrollo sostenible y la formación investigadora, entre otros. En cada uno de ellos se señalan y proponen temáticas e interrogantes asumibles para aportar un conocimiento científico educativo valioso para la sociedad. Esta sistematización pretende orientar y animar a jóvenes investigadores a trabajar sobre cuestiones relevantes que constituyen un reto presente y futuro para la investigación educativa.

Palabras clave: Investigación Pedagógica; Tecnología Educacional; Desarrollo Sostenible; Formación de Investigadores.

\footnotetext{
Correspondencia: Mํㅗ Pilar Colás-Bravo, pcolas@us.es. Facultad de CC de la Educación. Universidad de Sevilla C/ Pirotecnica s/n 41013 Sevilla (España).

1 Esta aportación es resultado de los siguientes Proyectos I+D subvencionados en convocatorias competitivas: Proyecto Europeo ERASMUS+ Designing For Personalization And Inclusion With Technologies. Referencia: 2017-1-IT02-KA201-036605. Fechas de ejecución: 2017-2020; Proyecto de Investigación Básica y Aplicada en Ciencias Sociales. Contrato de financiamiento № IBA-CS-10-2020- UNSA (Perú). Convocatoria 2019-2022. Y del Proyecto financiado por el Ministerio de Ciencia, Innovación y Universidades (España) RED2018-102439-T. Fechas de ejecución: 2020-2022.
} 


\begin{abstract}
This paper provides a wide view of the challenges of educational research in a current context characterized by the COVID 19 pandemic at the international level. It is based on the keynote lecture given on the occasion of the 2020 Ibero-American Award for Excellence in Educational Research awarded to the author of this article. The analysis of the social, economic, health and educational effects of the COVID 19 pandemic serves as a reference point to mark possible scientific trajectories of educational research. Areas of interest for new studies are ICT and education, sustainable development, and research training, among others. For each area, themes and attainable goals are pointed out and proposed to contribute valuable educational scientific knowledge to society. This systematization aims to guide and encourage young researchers to work on relevant issues that constitute a present and future challenge for educational research.

Keywords: Educational Research; Educational Technology; Sustainable Development; Research Training.
\end{abstract}

\title{
Introducción
}

Esta aportación forma parte de la Lección Magistral que tuve el honor de impartir con motivo de la concesión del Premio Iberoamericano a la Excelencia en Investigación Educativa 2020, que me fue otorgado por la Red Iberoamericana para el Desarrollo y difusión de la Investigación Educativa, en la ciudad de México.

Con tal motivo se me propuso que planteara los retos de la investigación educativa para el presente siglo. Si bien esta propuesta es, desde mi punto de vista excesivamente ambiciosa y, honestamente inabordable, considero que la situación de crisis sanitaria provocada por la actual pandemia COVID 19, está marcando caminos urgentes de abordaje desde la perspectiva de la investigación educativa. En este nuevo escenario surgen cuestiones importantes sobre las que se necesitan nuevos conocimientos. Por ello, nuestro propósito es poner de relieve líneas de trabajo que sirvan de referencia para jóvenes investigadores que necesitan iniciar su carrera investigadora. Pero también señalar caminos que son relevantes para dar respuesta a los retos de la sociedad.

La conexión entre países de habla hispana es una oportunidad para converger en tareas científicas y así avanzar en conocimientos científicos educativos contextualizados. En este sentido nuestro propósito, es apuntar y/o plantear cuestiones que sirvan de punto de partida para iniciar y desarrollar caminos fructíferos en la investigación educativa.

Pero ¿cuáles son los retos actuales de la investigación educativa? Identificarlos de manera pormenorizada y certera, es difícil y complejo. No obstante, este nuevo contexto social, generado y marcado por la pandemia, vislumbra la relevancia de determinadas líneas de investigación que suponen cambios sustantivos en la educación y en el conocimiento científico sobre ella.

Las transformaciones sociales exigen una reconducción de los caminos iniciados. Un claro ejemplo lo tenemos en la investigación médica. La investigación en estos momentos se ha focalizado en el estudio del COVID 19 y sus variantes. Numerosos grupos de investigación abordan desde distintas perspectivas el estudio de este virus porque urge encontrar vacunas que permitan inmunizar a la población. La ciencia se 
pone al servicio de la sociedad y se convierte en herramienta clave para superar esta crisis sanitaria, de carácter internacional.

Pero esta pandemia ha afectado y trastocado otros sectores, tales como la economía, la sociedad y con ella a la educación. Estamos ante un panorama nuevo que debe ser analizado, para encontrar soluciones creativas que posibiliten superar esta gran crisis mundial.

En este sentido podemos avanzar que la situación actual, marcada por la pandemia originada por el COVID 19, ha generado cambios sustantivos a nivel educativo, que requieren de nuevos conocimientos. Concretamente ha supuesto la irrupción de manera inmediata y extensiva de las TIC en los sistemas educativos. Si antes, estas, se entendían como herramientas básicamente de apoyo para la enseñanza y el aprendizaje, el COVID 19 las ha puesto en primer plano, convirtiéndose en el medio esencial e insustituible para la enseñanza en todos los niveles educativos. Ello ha creado toda una serie de problemáticas que requieren ser investigadas. A este referente se añade otro, no menos importante, que es la sostenibilidad de los sistemas vigentes.

La sostenibilidad es otro aspecto que se hace patente. Los sistemas sanitarios se manifiestan insostenibles para atender la enorme demanda sanitaria provocada por la pandemia. Y quedan patentes déficits en los sistemas económicos y sociales vigentes, que repercuten y afectan a numerosos colectivos, generando desigualdad y pobreza. Los cambios en los hábitos socioculturales y comunicativos también están teniendo consecuencias a nivel psicológico y mental, afectando al bienestar de las personas. Todos estos aspectos son claves, al considerarlos desde la perspectiva de la sostenibilidad económica y social.

Otro aspecto que se pone de manifiesto es el valor y la mirada esperanzada hacia la investigación. La investigación pasa a primer plano y los medios de comunicación divulgan este conocimiento. La tarea científica aumenta su visibilidad respecto a periodos anteriores. Es la expresión de un cambio de modelo, en el que la ciencia se presenta como la solución a problemas sociales. Pero también es el contexto idóneo en el que cobra especial significado el concepto de transferencia del conocimiento. Los resultados científicos deben ser conocidos, transmitidos y aplicados a la sociedad.

Estos tres referentes, TIC, desarrollo sostenible y formación científica, sirven como coordenadas para plantear retos actuales y futuros de la investigación educativa. Somos conscientes de que este desarrollo no agota otros posibles análisis y enfoques que, sin duda, ayudarían a enriquecer y nutrir de ideas a los investigadores educativos.

Remitiéndome a un paralelismo pictórico, esta exposición la hacemos a modo "puntillista", en el sentido de que con pequeños puntos o marcadores podamos crear un paisaje. Las líneas marcadas actúan a modo de perspectiva sobre las que crear la imagen de la investigación educativa, que es necesario construir.

\section{TIC y educación}

En lo que respecta a la educación real, la pandemia COVID 19 ha sido un fenómeno claramente disruptivo, en tanto que ha generado y obligado a depender de las tecnologías en la educación, además de en otros muchos sectores. Si antes, estas, se entendían como herramientas básicamente de apoyo para la enseñanza y el aprendi- 
zaje, el COVID 19 las ha puesto en primer plano, convirtiéndose en el medio esencial e insustituible para la enseñanza. La necesidad de confinamiento en 2020 generó que los hogares se convirtiesen en obligados espacios educativos y las TIC mediaron la interacción didáctica entre docentes y discentes. Esta situación de cambio radical, de una enseñanza presencial a una enseñanza online, genera numerosos interrogantes para la investigación educativa, por los numerosos efectos que ha tenido y que todavía hoy desconocemos.

A pesar de que la investigación sobre TIC y Educación cuenta con una larga trayectoria de aportaciones, y también hallazgos y descubrimientos, también es cierto que debido a este nuevo escenario emergen inesperadas problemáticas que constituyen retos para la investigación educativa. Por tanto, algunos interrogantes se hacen patentes en el momento actual, tales como:

¿Cómo afecta la enseñanza online, en los distintos niveles educativos, a los procesos de aprendizaje? ¿Se aprende lo mismo que con la enseñanza presencial? Dado que se están incorporando diversidad de recursos tecnológicos a los aprendizajes y que este se convierte en multimodal, cabe pensar que este cambio tecnológico está generando procesos cognitivos distintos a los que se reforzaban a través de procedimientos formativos tradicionales. Por tanto, en este sentido, es importante indagar sobre los efectos cognitivos en el aprendizaje según niveles escolares.

Desde el punto de vista del alumnado cabría preguntarse: ¿Qué nuevas dificultades en el aprendizaje se generan? ¿En qué medida la reducción de la socialización educativa, habitual en los espacios de aula, afecta al aprendizaje? Es una cuestión muy relevante, tanto a nivel científico como práctico. La observación directa de esta situación nos hace aventurar que se produce una desestabilización personal generada por la pérdida de referentes interiorizados, tales como el docente y los compañeros de clase.

En el plano docente, las competencias docentes adquieren especial protagonismo, y surge el interrogante: ¿Qué competencias digitales tienen y/o son necesarias que el docente domine para desenvolverse en la formación online en la enseñanza básica y secundaria? ¿Y en la universitaria? Esta temática ocupa la atención de la investigación y publicaciones recientes (Amhag et al., 2019; Arango et al., 2020; Fraile et al., 2018), además de modelos desarrollados por organismos internacionales que marcan las líneas maestras de desarrollos futuros para la formación docente, tales como el modelo del Marco Europeo para la Competencia Digital del Profesorado (European Framework for the Digital Competence of Educators: DigCompEdu) (Comisión Europea, 2017). Sin embargo, no contamos todavía con datos y conocimientos suficientes sobre esta temática, por lo que se convierte en un reto para la investigación educativa.

De forma similar, respecto a las habilidades tecnológicas del alumnado (a pesar de la visión extendida de que los jóvenes son nativos digitales), nos lleva al siguiente interrogante: ¿Qué competencias digitales discentes son necesarias desarrollar? Esta cuestión se puede abordar desde dos ángulos: uno, entendidas como objetivos de aprendizaje, es decir, competencias digitales a desarrollar a través de los sistemas educativos, o como recurso para su aprendizaje, y por tanto capacidad para moverse y aprender a través del formato de enseñanza online (Martzoukou et al., 2020). También contamos con aportaciones científicas valiosas respecto a la formación en competencias digitales como parte de la educación ciudadana (Guitert et al., 2020), que abarcan desde a alfabetización digital, 
al pensamiento computacional (Nouri et al., 2020). Sin embargo, es importante avanzar y ampliar el conocimiento disponible en la actualidad, por lo que constituye una temática relevante para investigaciones venideras.

Otra dimensión importante que incorporar en la investigación educativa, relacionada con las TIC, es el aspecto emocional.

¿Qué consecuencias emocionales genera una enseñanza online? ¿Aumenta la sensación de soledad? ¿Disminuye el bienestar? ¿Qué efectos tiene respecto a la implicación y compromiso de los estudiantes con sus estudios? Esta dimensión emocional está siendo cada vez más importante y acaparando la atención de numerosos estudios (Collazos et al., 2021; Tempelaar et al., 2012; Wortha et al., 2019). Si bien, años atrás, no se contemplaba en la comprensión de los procesos de aprendizaje, en la actualidad se considera que es una dimensión que afecta y regula de manera importante el aprendizaje. En una investigación realizada por mi equipo de investigación en la década de 2010 (De Pablos-Pons et al., 2013) descubrimos que la implicación y el compromiso está estrechamente relacionado con el bienestar y la felicidad. Si las tecnologías son elementos motivadores cabe esperar que aumentará la implicación y el compromiso de los estudiantes, pero esta hipótesis está todavía por contrastar. Por tanto, y como síntesis, es una línea de trabajo de gran potencial para comprender y dar respuesta a problemáticas educativas actuales.

Las dificultades de acceso a Internet por parte de la población escolar, pone de relieve y en primer plano el concepto de brecha digital. Este fenómeno tiene efectos y consecuencias notables, tanto en la escolarización, como en el abandono escolar. Por tanto, su abordaje desde la perspectiva de la investigación educativa es importante. Desde los gobiernos se están tomando medidas para reducir la brecha digital a través de fuertes inversiones en infraestructuras (Ministerio de Educación et al., 2020). También contamos con estudios estadísticos que dan fe de variables, de carácter externo, que afectan a la brecha digital, tales como, nivel socioeconómico, zonas geográficas, género, edad, etc. (Rogerson, 2020; Vasilescu et al., 2020). Sin embargo, son necesarias aportaciones que incluyan variables educativas relevantes, tales como niveles de logro académico, desarrollo intelectual, éxito y abandono escolar, entre otras. La otra cara de la moneda la constituye la inclusión educativa, que se puede propiciar con las TIC (Colás-Bravo et al., 2019). Por tanto, queda patente, como reto, dilucidar, a través de la investigación educativa, el potencial y los efectos de las TIC en cuanto a exclusión o inclusión educativa.

Desde estas coordenadas planteamos algunos interrogantes que pueden orientar líneas fructíferas de investigación en la educación:

¿Cómo afecta la enseñanza online a los procesos de aprendizaje?

¿Qué nuevas dificultades en el aprendizaje se generan cuando la enseñanza se lleva a cabo mediante formación online en todos los niveles educativos?

¿Qué competencias digitales tienen y/o son necesarias que el docente domine?

¿Qué competencias digitales discentes son esenciales desarrollar? ¿Cuáles están ya logradas?

¿Qué consecuencias emocionales produce una enseñanza online, en cuanto a la motivación, interés e implicación del alumnado?

¿Qué variables relacionadas con las TIC derivan en exclusión y/o brecha digital?

¿Qué prácticas con TIC han demostrado ser facilitadoras de inclusión? 


\section{Educación para un mundo sostenible}

Un reto que marca tanto la investigación educativa, en el presente como en el futuro, es la sostenibilidad. La sostenibilidad es una meta formulada por Naciones Unidas y planteada como un reto en la AGENDA 2030.

Por sostenibilidad, a nivel general, entendemos la capacidad de preservar los recursos y adoptar fórmulas para afrontar las condiciones cambiantes. Se trata en definitiva de satisfacer las necesidades del presente, sin poner en juego, ni comprometer, las condiciones de vida de las futuras generaciones. El desarrollo sostenible se orienta a elevar los niveles de bienestar de las sociedades humanas de hoy, además de preservar para las generaciones futuras un mundo, con aceptables niveles de salud ambiental, económica y social.

Los programas y líneas de actuación en sostenibilidad abarcan cuatro ámbitos; el ambiental, el económico, el social y el educativo.

(1) La sostenibilidad ambiental, se refiere a la necesidad de preservar los ecosistemas y conservarlos para las generaciones venideras.

(2) La sostenibilidad económica, entendida como un crecimiento económico, inclusivo y respetuoso con el medio ambiente. Es decir, promover un crecimiento económico que genere riqueza equitativa para todos, sin dañar el medio ambiente.

(3) La sostenibilidad social, implica la necesidad de garantizar el bienestar social, la inclusión y la equidad.

La sostenibilidad social, se proyecta al logro de la reducción de las desigualdades, educación inclusiva, la equidad de género, la educación de calidad, la alfabetización universal, la ciudadanía responsable y activa, promover la paz y la justicia diversidad e inclusión social y cultural y el acceso a un empleo digno y al emprendimiento.

(4) La sostenibilidad educativa se articula en torno a la equidad de género, acceso a la formación, inclusión educativa, alfabetización universal, empleo y emprendimiento. Estas referencias generan múltiples temáticas y metas de investigación que conciernen a la investigación educativa presente y futura.

Algunas de estas temáticas forman parte de líneas de investigación que en la actualidad mantienen una actividad estimable (Cavus et al., 2021; Colás-Bravo et al., 2017). Nos referimos a las temáticas de género y educación, inclusión educativa y atención a la diversidad, así como empleo y emprendimiento. En estos casos se pretende encontrar y experimentar fórmulas pedagógicas para reforzar el desarrollo sostenible en estas orientaciones. En el primer caso prima la investigación que pretende mostrar y visibilizar la situación actual de discriminación por motivo de género en diferentes contextos; social, educativo, económico, gobernanza, etc. En cuanto a la línea de inclusión educativa y atención a la diversidad, las investigaciones actuales se orientan a diagnosticar necesidades educativas y a desarrollar propuestas pedagógicas que favorezcan la inclusión. Tienen cabida en esta línea la producción científica sobre multiculturalidad, brecha digital y abandono escolar. Y por último el empleo y emprendimiento es una línea de trabajo que se impulsa y apoya a través de programas $\mathrm{I}+\mathrm{D}$, a raíz de la problemática del fuerte desempleo provocado por las crisis económicas. 
Por tanto, a modo de síntesis, podemos decir que las directrices marcadas por la AGENDA 2030 por Naciones Unidas, han forjado y consolidado temáticas relevantes sobre las que proyectar la investigación educativa presente y futura.

Pero desde mi punto de vista, la conciencia sostenible, es la que posibilita activar y avanzar en el logro de los sistemas sostenibles; económicos, ambientales, sociales y educativos. Es la conciencia la que conduce o lleva a la acción responsable para operar en todos los ámbitos de la sostenibilidad. Pero esta no se alcanza de forma automática, sino que exige unos procesos previos de aprendizaje fundamentados en conocimientos, valores y actitudes. No obstante, aunque estos son necesarios, no son suficientes por si mismos para lograr la conciencia sostenible, ya que ésta implica niveles de pensamiento de naturaleza superior. La conciencia es el nivel más alto de aprendizaje vital (Colás-Bravo et al., 2018). Esa conciencia permite obrar activamente en los ámbitos económicos, sociales, ambientales y educativos (Schniederjans, \& Khalajhedayati, 2021). Un ejemplo del valor de la conciencia para la acción social lo tenemos en estos momentos en la situación de pandemia actual, en la que se hace una referencia constante a la necesidad de una conciencia ciudadana para actuar responsablemente. Pues este es el quid de la cuestión, ¿cómo desarrollar la conciencia ciudadana? Uno de los autores más representativos, a nivel educativo, que recurre al concepto de conciencia como eje clave de la educación es Freire (1985). Según él, la educación consiste en la concientización, es decir, en el desarrollo de una toma de conciencia crítica, que tiene lugar a partir del análisis e interpretación de la realidad. Este autor distingue tres tipos de conciencia, aplicables tanto para el análisis de la evolución personal, como para el estudio de grupos sociales: conciencia mágica, conciencia ingenua y conciencia crítica. Cada una de ellas conlleva actitudes y modos cognitivos de pensar diferentes. Para este autor, el desarrollo de la conciencia es un proceso que incluye tres estadios o fases: sensibilización, toma de conciencia crítica y acción transformadora. Y conlleva una evolución a través de la interacción y reflexión sobre la práctica, que conduce a una interpretación más profunda de la realidad y a nuevos niveles de comprensión. Investigaciones educativas han detectado diferentes niveles de conciencia en el profesorado en formación sobre su práctica docente (Colás-Bravo et al., 2018).

En primera instancia, la incorporación de contenidos curriculares sobre sostenibilidad (ambiental, económica, social y educativa) en los programas formativos son iniciativas necesarias para crear una base de conocimientos. En estos saberes se sustentan las actitudes y competencias sostenibles. Y estas, son a su vez, los cimientos en los que asentar la conciencia y por tanto la acción responsable. Por ejemplo, la incorporación de programas de doctorado sobre equidad de génerocontenidos y conocimientos que forman parte de la sostenibilidad-constituyen el soporte, tanto para cimentar una toma de conciencia sobre esta problemática social, como para propiciar acciones y proyectos en pro de la equidad. A través de esta propuesta formativa se puede generar una conciencia que conduce a una acción responsable (conciencia sostenible). Esa conciencia permite obrar activamente en los ámbitos económicos, sociales, ambientales y educativos. En la siguiente figura se representan gráficamente estas ideas. 


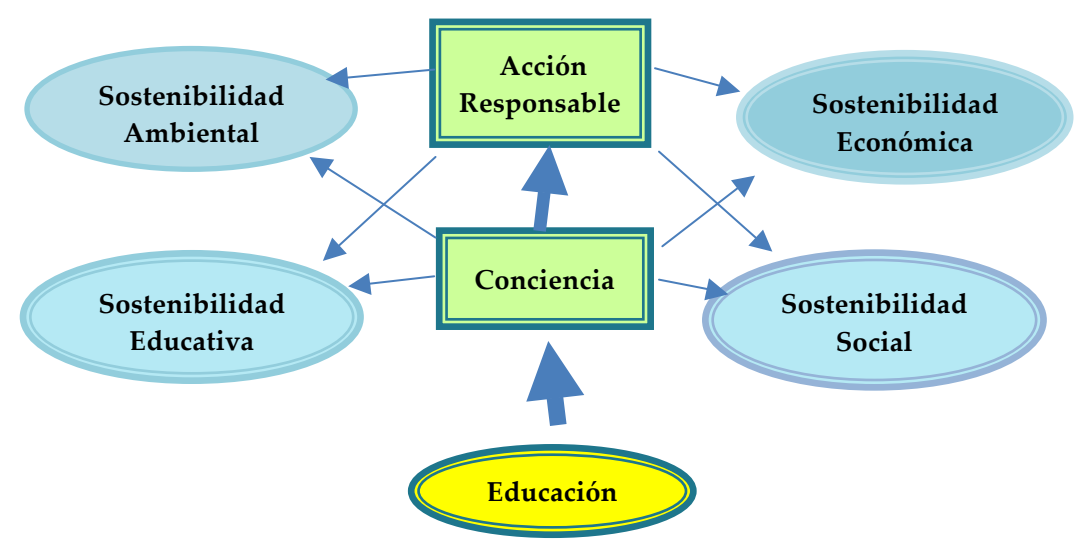

Figura 1. Retos de la Investigación Educativa respecto a la Sostenibilidad. Elaboración propia.

Por tanto, desde la perspectiva del desarrollo sostenible, en la actual situación de COVID, se pueden plantear algunos interrogantes tales como:

¿Cómo activar la conciencia ciudadana para un comportamiento responsable ante la situación de COVID 19?

¿Qué papel juega el conocimiento y la educación en los comportamientos responsables?

¿Ha cambiado esta pandemia el pensamiento y conocimiento de la realidad, desde una perspectiva sostenible?

¿Cómo afecta la pandemia a la calidad educativa? ¿Se ha reducido o ha aumentado el abandono escolar?

Todos estos interrogantes pueden iluminar proyectos de investigación educativa útiles desde la perspectiva de la sostenibilidad en la actual situación de pandemia a escala mundial.

No obstante cuestiones de mayor calado pueden formar las agendas de investigación para el futuro. En este sentido desde la perspectiva del desarrollo sostenible, se pueden plantear algunos interrogantes para canalizar el futuro de la investigación:

¿Cómo conseguir este cambio de pensamiento, actitudes y prácticas sociales a través de la Educación?

¿Qué formación debe tener el profesorado para educar a los estudiantes en comportamientos responsables sostenibles?

¿Qué prácticas de enseñanza son más eficaces y valiosas, para alcanzar una ciudadanía responsable en todos los ámbitos vitales?

¿Qué papel juegan las TIC en el desarrollo de competencias sostenibles?

¿Cómo afecta la educación a los indicadores de pandemia (muertes y contagios)? 
Ya contamos con algunas aportaciones en esta línea (Morales-López et al., 2021). En este estudio se establece como hipótesis de trabajo la relación entre muertes por COVID y nivel de formación educativa. Los resultados muestran que el $71,2 \%$ de mortalidad se da en personas que tienen niveles de estudio hasta educación básica, el $15,17 \%$ en los sujetos con estudios medios y el 13, 1\%, con estudios superiores. Estos datos se obtienen del Instituto Nacional de Estadística y Geografía de México. Este estudio concluye que la Educación es uno de los factores fundamentales en el peso que tiene la mortalidad por COVID 19. Estos datos podrían ser contrastados y replicados a través de estudios similares en otros países.

En síntesis, si la sostenibilidad constituye uno de estos retos fundamentales de la sociedad actual para los años venideros, es preciso dotar al conjunto de agentes educativos, especialmente al profesorado, de herramientas conceptuales y metodológicas que posibiliten una educación para la conciencia y acción responsable de la ciudadanía en todos los ámbitos de la sostenibilidad. Y para ello es necesario el desarrollo de conocimiento científico-pedagógico derivado de la investigación.

Este es un camino acorde con los ODS (objetivos de desarrollo sostenible) y la agenda 2030 de Naciones Unidas, respecto a la Educación para Desarrollo Sostenible. En concreto, con su Programa de Acción Mundial (GAP) para el Desarrollo Sostenible, en relación con la Educación (ODS 4), se pretenden alcanzar dos claros objetivos:

1) Reorientar la educación y el aprendizaje para que todos tengan la posibilidad de adquirir los conocimientos, las competencias, los valores y las actitudes necesarias para contribuir al desarrollo sostenible;

2) Fortalecer el papel de la educación en todos los planes de acción, programas y actividades para promover el desarrollo sostenible (UNESCO, 2015, p. 14).

Para conseguir la meta del desarrollo sostenible deseado es condición necesaria e imprescindible una educación basada en la formación de un pensamiento y conciencia que permita una acción responsable de la ciudadanía en todos los ámbitos de la sostenibilidad. Todos estos cambios solo serán posibles desde una educación que promueva la responsabilidad y la conciencia social de la ciudadanía para que puedan tomar decisiones que favorezcan la calidad de vida, objetivo de la sostenibilidad.

\section{Formación investigadora}

Una tercera faceta que, a nuestro entender, debe ser seriamente considerada desde la investigación educativa es la referida a la formación investigadora. Una de las consecuencias que ha generado esta situación de crisis pandémica es la percepción del gran valor social de la investigación. Sin embargo, es poca la producción científica sobre esta temática, exceptuando ámbitos geográficos específicos, como es el caso de Australia. La formación investigadora es un área por excelencia eminentemente pedagógica, que hoy por hoy, no ha recibido suficiente atención. En este sentido Rojas-Betancur (2009) reconoce que uno de los grandes problemas de las instituciones de educación superior para potenciar la producción científica es la formación y retención de nuevos investigadores. Si bien existe una buena actitud hacia la investigación por parte de los 
graduados jóvenes, existen grandes dificultades coyunturales y estratégicas para lograr tal propósito, además de escasa contrastación de modelos de formación investigadora. Este autor concluye que la formación de jóvenes investigadores constituye un problema esencialmente pedagógico, porque las instituciones universitarias poseen poca capacidad para sistematizar acciones destinadas a la formación y el entrenamiento científico. Esta manifiesta necesidad exige un abordaje educativo, implicando y conjugando modelos pedagógicos, metodologías didácticas, así como competencias formativas a desarrollar, entre otros muchos aspectos.

En convergencia con esta aproximación, en la formación investigadora son importantes, a nuestro entender, tres áreas de desarrollo pedagógico, que deben estar fundamentadas en el conocimiento previo generado por la investigación:

a) Identificación de enfoques pedagógicos (conductual, constructivista, sociocultural, etc.) que subyacen en las enseñanzas de los programas de doctorado) y contrastación de su valor para el aprendizaje.

b) Modelos de formación investigadora existentes. Ya contamos con aportaciones incipientes (Colás Bravo, et al., 2014; De Pablos et al., 2015) que identifican tres modelos teóricos existentes: técnico, personalista y cultural. Cada uno de ellos plantea la formación investigadora de forma distinta, incidiendo en enseñanzas diferenciadas, tanto en lo referente a competencias a desarrollar, como en prácticas recomendables para alcanzarlas.

c) Diagnóstico y evaluación de competencias investigadoras.

d) Identificación de buenas prácticas de pedagogía investigadora.

De estas áreas de indagación podrían derivarse los siguientes interrogantes de investigación:

¿Cómo debe ser la formación investigadora?

¿Cuáles son los modelos óptimos que lleven a la excelencia investigadora?

¿Qué competencias son esenciales para formar a investigadores exitosos?

Dado el protagonismo e insistente mirada de la sociedad hacia los logros y hallazgos científicos (en este momento concretamente hacia la medicina), es una oportunidad para tomar conciencia de la importancia de considerar la formación investigadora como un área importante de exploración pedagógica. Es precisamente en el campo de la medicina donde existe mayor producción científica sobre esta cuestión (Chambers et al., 2016), aportando conocimientos valiosos, tanto en cuanto a los modelos de formación, como respecto a las competencias. Sin embargo, urge la indagación pedagógica sobre esta área formativa, tan clave para la sociedad.

En España contamos con algunas aproximaciones respecto a la formación doctoral en lo que respecta a modelos formativos y diagnóstico de competencias investigadoras (Colás-Bravo \& Hernández de la Rosa, 2021; Colás-Bravo et al., 2014; De Pablos et al., 2015). Sin embargo, entendemos que es preciso impulsar estudios que ayuden a identificar y descubrir claves pedagógicas para la formación investigadora. Todo un mundo nuevo por descubrir y sistematizar. 
Por otra parte, el impulso institucional, dado por la Administración Pública, a la transferencia de la investigación, genera otro nuevo campo de competencias investigadoras que es necesario dilucidar y clarificar. Ya existen algunas aportaciones que tratan de identificar necesidades de formación en investigación, en cuanto a la difusión e implementación de los resultados de la investigación (Tabak et al., 2017). Sin embargo, es una temática que no se aborda ni se investiga al nivel que sería necesario desde su vertiente pedagógica.

Las redes de investigación educativa ya creadas, tanto a nivel nacional, como la Red de Investigación educativa AIDIPE, y otras más recientes, a nivel iberoamericano, como la Red Iberoamericana para el Desarrollo y Difusión de la Investigación Educativa, son espacios idóneos para impulsar la investigación tanto sobre la educación como sobre la formación investigadora. Se precisan nuevos conocimientos sobre cuestiones tales como:

¿Cuáles son los programas formativos de doctorado más efectivos para potenciar investigadores de excelencia?

¿Qué metodologías de acompañamiento tutorial son las más exitosas en la formación investigadora?

¿Cuáles son las competencias investigadoras clave a desarrollar en los programas de doctorado?

¿Cómo evaluar la calidad científica de los investigadores?

¿Cómo se construye la identidad como investigador/a?

¿Cómo se producen los procesos de aculturación científica?

¿Cuáles son los aportes de las redes profesionales de investigación a la formación investigadora?

¿Qué dificultades debe superar una mujer para incorporarse al campo de la investigación educativa?

¿Qué relaciones interpersonales virtuales son más provechosas y efectivas en los procesos de formación de investigadores?

¿Qué aprendizajes y mecanismos gramaticales, sintácticos y lógicos son esenciales dominar para la escritura científica?

¿Cuál es el acompañamiento académico y emocional apropiado para una formación investigadora de calidad?

¿Cuál es el valor social, económico y cultural que aporta la investigación?

Es creciente el interés por estas temáticas en países latinoamericanos, llevados por la idea de que la formación de investigadores es clave, para el impulso y la consolidación de la investigación en la universidad, pero también para el desarrollo social y económico de los países.

La formación investigadora requiere de una formación equilibrada respecto al ámbito cognoscitivo de la disciplina, metodológico, cultural, epistemológico y personal. Por ello se precisa de investigaciones que abarquen estas dimensiones, para aportar conocimiento científico educativo, valioso por su aplicabilidad a la actual formación investigadora de todos los campos disciplinares. 


\section{Un futuro en construcción}

El propósito de esta aportación es identificar líneas y áreas relevantes para la investigación educativa, en base a las nuevas condiciones y necesidades sociales marcadas por la pandemia. Tres ejes han servido a este propósito: El presente, marcado por la formación online; las directrices de desarrollo sostenible propuestas por organismos internacionales; y la revalorización del papel de la investigación y del conocimiento científico en su contribución al bienestar social. Desde estos referentes, se detectan tres espacios relevantes que urge su abordaje. La conjunción de ellos sirve de base para crear un escenario en el que visibilizar los retos de la investigación educativa presente y futura, como puede verse en la figura 2.

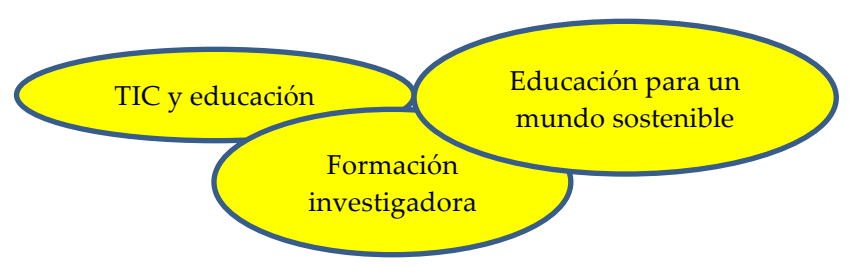

Figura 2. Áreas que constituyen retos para la investigación educativa; presente y futura. Elaboración propia

Pero estos caminos se están iniciando. Así, por ejemplo, en el caso de las políticas educativas en materia de investigación y desarrollo sostenible, en 2021, en España, los Presupuestos Generales del Estado incrementan en un $89 \%$ los gastos en I+D respecto a los últimos años. Dato que es un claro indicador de la revalorización de la investigación a raíz de la pandemia. Respecto a la tecnología educativa, se ha lanzado el programa Educa en Digital (Ministerio de Educación y Formación Profesional, 2020) con el objetivo de apoyar la transformación digital de la educación en España. Las actuaciones comenzaron a ejecutarse durante el primer trimestre del curso 2020-2021. Educa en Digital nace de la necesidad de dar respuesta a una realidad que la emergencia sanitaria del COVID-19 ha puesto de manifiesto, que el sistema educativo precisa de un impulso en la digitalización. La disponibilidad de recursos tecnológicos e infraestructuras son determinantes a la hora de reducir posibles brechas digitales y promover la cohesión social. De ahí que este programa prevea la dotación de medio millón de dispositivos con conectividad a centros educativos que pondrán a disposición de los estudiantes para impulsar la educación digital. Esta iniciativa pretende paliar los efectos de la brecha digital en el alumnado, y especialmente el que se encuentra en situación vulnerable, motivada por la no disponibilidad de dispositivos, conectividad o herramientas adecuadas para poder seguir los procesos de aprendizaje en un entorno digital, tanto de forma presencial en los centros educativos, como desde el hogar.

La situación de emergencia sanitaria no sólo ha afectado al alumnado; el cuerpo docente ha tenido que llevar a cabo un extraordinario esfuerzo de adaptación a un 
escenario de enseñanza a distancia que requiere de competencias y recursos digitales. Por ello, también se ponen en marcha iniciativas que van dirigidas a la capacitación docente en competencias digitales. En este sentido, el Ministerio de Educación y Formación Profesional, a través del Instituto Nacional de Tecnologías Educativas y Formación del Profesorado (INTEF), ha multiplicado la oferta de materiales y herramientas para la educación digital, así como la de cursos de capacitación docente para adecuar las competencias del profesorado a la educación en un entorno digital. Este esfuerzo también lo han realizado las universidades, en su ámbito de autonomía.

Junto a estas iniciativas, la realidad también muestra y señala caminos relevantes para una investigación educativa fructífera. Por ejemplo, la violencia de género es, entre otros, un ejemplo de problemática tanto contextual como global, que necesita de un abordaje educativo, pero también la actividad investigadora de las mujeres, o la educación para la salud en tiempos de pandemia, entre otros.

Confío y tengo esperanza, que el propósito de este texto, que es servir de orientación a jóvenes investigadores sobre áreas relevantes de investigación que les ayude a focalizar sus futuras líneas de investigación, cumpla con el cometido con el que fue gestado.

\section{Referencias}

Amhag, L., Hellström, L., \& Stigmar, M. (2019). Teacher educators' use of digital tools and needs for digital competence in higher education. Journal of Digital Learning in Teacher Education, 35(4), 203-220. http://dx.doi.org/10.1080/21532974.2019.1646169

Arango, D., Fernández, J., Rojas, O., Gutiérrez, C., Villa, C., y Grisales, M. (2020). Competencia digital en docentes universitarios: Evaluación de relación entre actitud, formación y alfabetización en el uso de TIC en entornos educativos. Revista Ibérica De Sistemas e Tecnologias De Informação, 538-552. https://search.proquest.com/scholarly-journals/competencia-digital-en-docentes-universitarios/docview/2394537952/ se-2 ?accountid=1474

Cavus, N., Sani, A., Haruna, Y. \& Lawan, A. (2021) Efficacy of social networking sites for sustainable education in the era of COVID-19: A systematic review. Sustainability, 13(2), 808. http://dx.doi.org/10.3390/su13020808

Colás Bravo, P. y Hernández de la Rosa, M. (2021). Las competencias investigadoras en la formación universitaria. Revista Universidad y Sociedad, 13(1), 17-25. https://rus. ucf.edu.cu/index.php/rus/article/view/1891.

Colás-Bravo, P., Giuseppe Rossi, P., de Pablos-Pons, J., Conde-Jiménez, J., y Villaciervos- Moreno, P. (2019). Aplicaciones digitales para la inclusión. El proyecto europeo DEPIT. Revista de Comunicación de La SEECI, 50, 169-192. https://doi.org/10.15198/ seeci.2019.50.169-192

Colás-Bravo, P., Magnoler, P., \& Conde-Jiménez, J. (2018). Identification of Levels of Sustainable Consciousness of Teachers in Training through an E-Portfolio. Sustainability 10(10), 3700. https://doi.org/10.3390/su10103700

Colás-Bravo, P., Reyes-de Cózar, S., y Conde-Jiménez, J. (2017). Efectos de las prácticas inclusivas en los centros educativos y su impacto en el estado emocional del profesorado. Polyphōnía. Journal of Inclusive Education, 1(2), 34-55. https://www.revista. celei.cl/index.php/PREI/article/view/233 
Colás-Bravo, P, González-Ramírez, T y Conde-Jiménez, J (2014). La formación investigadora (I). Modelos pedagógicos. Universidad de Barcelona. http://hdl.handle.net/2445/53640 Collazos, C., Fardoun, H., AlSekait, D., Pereira, C. \& Moreira, F. (2021). Designing online platforms supporting emotions and awareness. Electronics, 10(3), 251. http:// dx.doi.org/10.3390/electronics10030251

Comisión Europea (2017). European Framework for the Digital Competence of Educators (DigCompEdu). https://ec.europa.eu/jrc/en/digcompedu.

Chambers, D., Proctor, E., Brownson, R. \& Straus, S (2016). Mapping training needs for dissemination and implementation research: lessons from a synthesis of existing D\&I research training programs. Translational Behavioral Medicine. http://dx.doi.org/10.1007/ s13142-016-0399-3

De Pablos-Pons, J., Colás-Bravo, P., González-Ramírez, T. \& Camacho Martínez-Vara del Rey, C. (2013). Teacher well-being and innovation with information and communication technologies; proposal for a structural model. Quality and Quantity 47, 2755-2767. http://dx.doi.org/10.1007/s11135-012-9686-3

De PablosPons, J., ColásBravo., GonzálezRamírez, T. y CondeJiménez, J. (2015). Dimensiones en las que fundamentar la formación investigadora en Tecnología Educativa. RELATEC Revista Latinoamericana de Tecnología Educativa, 14(1), 5773.

Fraile, M., Peñalva-Vélez, A., \& Mendióroz -Lacambra, A. (2018). Development of digital competence in secondary education teachers' training. Education Sciences, 8(3) http:// dx.doi.org/10.3390/educsci8030104

Freire, P. (1985). The Politics of Education: Culture, Power, and Liberation. Greenwood Publishing Group.

Martzoukou, K., Fulton, C., Kostagiolas, P. \& Lavranos, C. (2020). A study of higher education students' selfperceived digital competences for learning and everyday life online participation. Journal of documentation [online], 76(6), 1413-1458. https://doi. org/10.1108/JD-03-2020-0041

Ministerio de Educación y Formación Profesional (MEFP) (2020). Acuerdo de la Conferencia Sectorial de Educación sobre el marco de referencia de la competencia digital docente. «BOE» núm. 191, de 13 de julio de 2020, páginas 50638 a 50668 (31 págs.) https://www.boe.es/eli/es/res/2020/07/02/(2)

Ministerio de Educación y Formación Profesional, Ministerio de Asuntos Económicos y Transformación Digital, y Entidad Pública Empresarial (2020). Ejecución del programa «Educa en Digital» «BOE» núm. 189, de 10 de julio de 2020, páginas 50047 a 50071 (25 págs.) https://www.boe.es/diario_boe/txt.php?id=BOE-A-2020-7682

Morales López, J., Ramos-Martínez, L., e Irigoyen-Coria, A. (2021). Grado de escolaridad en México y su impacto sobre la mortalidad por Covid-19: una breve reflexión. Archivos en Medicina Familiar, 23(2) 63-65.

Nouri, J., Zhang, L., Mannila, L., \& Norén, E. (2020). Development of computational thinking, digital competence and century skills when learning programming in K-9. Education Inquiry, 11(1). https://search.proquest.com/scholarly-journals/developmentcomputational-thinking-digital/docview/2351042303/se-2?accountid=14744

Rogerson, S. (2020). The digital divide is a multi-dimensional complex. Journal of Information, Communication E Ethics in Society, 18(3), 321. http://dx.doi.org/10.1108/JICES05-2020-0060 
Rojas-Betancur, H. M. (2009). Formar investigadores e investigadoras en la universidad: optimismo e indiferencia juvenil en temas científicos. Revista Latinoamericana de Ciencias Sociales, Niñez y Juventud, 7(2), 1595-1618. https://www.redalyc.org/ pdf/773/77314999018.pdf

Schniederjans, D., \& Khalajhedayati, M. (2021). Competitive sustainability and stakeholder engagement: Exploring awareness, motivation, and capability. Business Strategy and the Environment, 30(2), 808-824. http://dx.doi.org/10.1002/bse.2655

Tabak, R., Padek, M., Kerner, J., Strange, K., Proctor, E., Dobbins, M., Colditz, G., Chambers, D., \& Brownson, R. (2017). Dissemination and implementation science training needs: Insights from practitioners and researchers. American journal of preventive medicine, 52(3), S322-S329.

Tempelaar, D., Niculescu, A., Rienties, B., Gijselaers, W. H., \& Giesbers, B. (2012). How achievement emotions impact students' decisions for online learning, and what precedes those emotions. Internet and Higher Education, 15(3), 161-169. http://dx.doi. org/10.1016/j.iheduc.2011.10.003

UNESCO (2015). Educación 2030. Declaración de Incheon: Hacia una educación inclusiva y equitativa de calidad y un aprendizaje a lo largo de la vida. http://www.unesco.org/new/ fileadmin/MULTIMEDIA/FIELD/Santiago/pdf/ESP-Marco-de-Accion-E2030-aprobado. pdf

Vasilescu, M., Serban, A., Dimian, G., Aceleanu, M., \& Picatoste, X. (2020). Digital divide, skills and perceptions on digitalisation in the european Union-Towards a smart labour market. PLoS One, 15(4). http://dx.doi.org/10.1371/journal.pone.0232032

Wortha, F., Azevedo, R., Taub, M., \& Narciss, S. (2019). Multiple negative emotions during learning with digital learning environments-Evidence on their detrimental effect on learning from two methodological approaches. Frontiers in Psychology, 10, 19. http:// dx.doi.org/10.3389/fpsyg.2019.02678

Fecha de recepción: 21 de febrero de 2021.

Fecha de revisión: 22 de febrero de 2021.

Fecha de aceptación: 15 de marzo de 2021. 